\title{
Estimation of Electronegativity Values of Elements in Different Valence States
}

\author{
Keyan Li, Dongfeng Xue* \\ State Key Laboratory of Fine Chemicals, Department of Materials Science and \\ Chemical Engineering, School of Chemical Engineering, Dalian University of \\ Technology, 158 Zhongshan Road, Dalian 116012, P. R. China \\ *Corresponding author. E-mail: dfxue@chem.dlut.edu.cn
}

\section{Supporting Information}

Some typical applications of the currently proposed EN scale, such as:

1. Estimation of the Lewis acid strength for the main group elements in their highest oxidation states (Figure S1 and Table S1).

2. Estimation of the hydration free energy of divalent metal ions for the first transition series (Figure S2 and Table S2).

In addition,

3. All the detailed data for calculating EN of six-coordinated cations and our calculated values (Table S3).

4. EN of trivalent $\mathrm{Ln}$ as a function of $\mathrm{CN}$ (Figure S3).

5. There is a fairly good relation between our current EN and Luo's EN (Figure S4 and Table S4). 


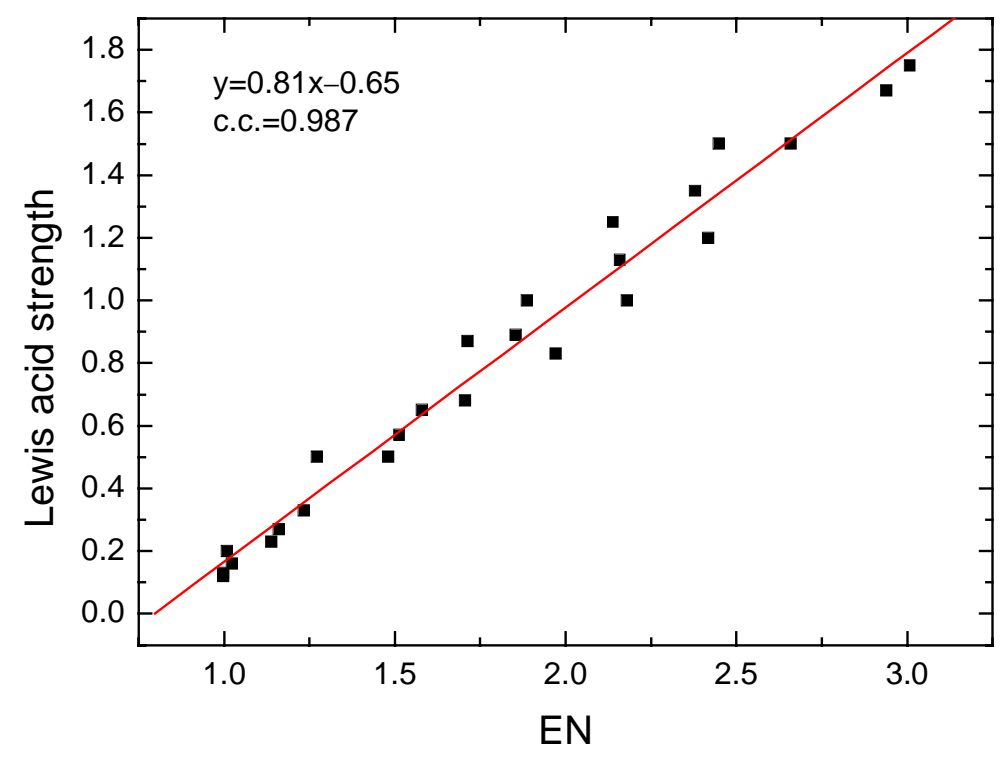

Figure S1. Correlation between Lewis acid strength and six-coordinated EN for main group elements in their highest oxidation state (values of EN and Lewis acid strength are given in Table $\mathrm{S} 1$ ). 
Table S1. The six-coordinated EN and Lewis acid strengths in valence units $(\mathrm{Sa})$

\begin{tabular}{cccccc}
\hline element & EN & $S a$ & element & EN & $S a$ \\
\hline $\mathrm{Li}$ & 1.009 & 0.20 & $\mathrm{Si}$ & 1.887 & 1.00 \\
$\mathrm{Na}$ & 1.024 & 0.16 & $\mathrm{Ge}$ & 1.854 & 0.89 \\
$\mathrm{~K}$ & 0.998 & 0.13 & $\mathrm{Sn}$ & 1.706 & 0.68 \\
$\mathrm{Rb}$ & 0.998 & 0.12 & $\mathrm{~N}$ & 2.939 & 1.67 \\
$\mathrm{Be}$ & 1.273 & 0.50 & $\mathrm{P}$ & 2.139 & 1.25 \\
$\mathrm{Mg}$ & 1.234 & 0.33 & $\mathrm{As}$ & 2.159 & 1.13 \\
$\mathrm{Ca}$ & 1.160 & 0.27 & $\mathrm{Sb}$ & 1.971 & 0.83 \\
$\mathrm{Sr}$ & 1.139 & 0.23 & $\mathrm{~S}$ & 2.659 & 1.50 \\
$\mathrm{~B}$ & 1.714 & 0.87 & $\mathrm{Se}$ & 2.448 & 1.50 \\
$\mathrm{Al}$ & 1.513 & 0.57 & $\mathrm{Te}$ & 2.180 & 1.00 \\
$\mathrm{Ga}$ & 1.579 & 0.65 & $\mathrm{Cl}$ & 3.008 & 1.75 \\
$\mathrm{In}$ & 1.480 & 0.50 & $\mathrm{I}$ & 2.417 & 1.20 \\
$\mathrm{C}$ & 2.380 & 1.35 & & & \\
\hline
\end{tabular}




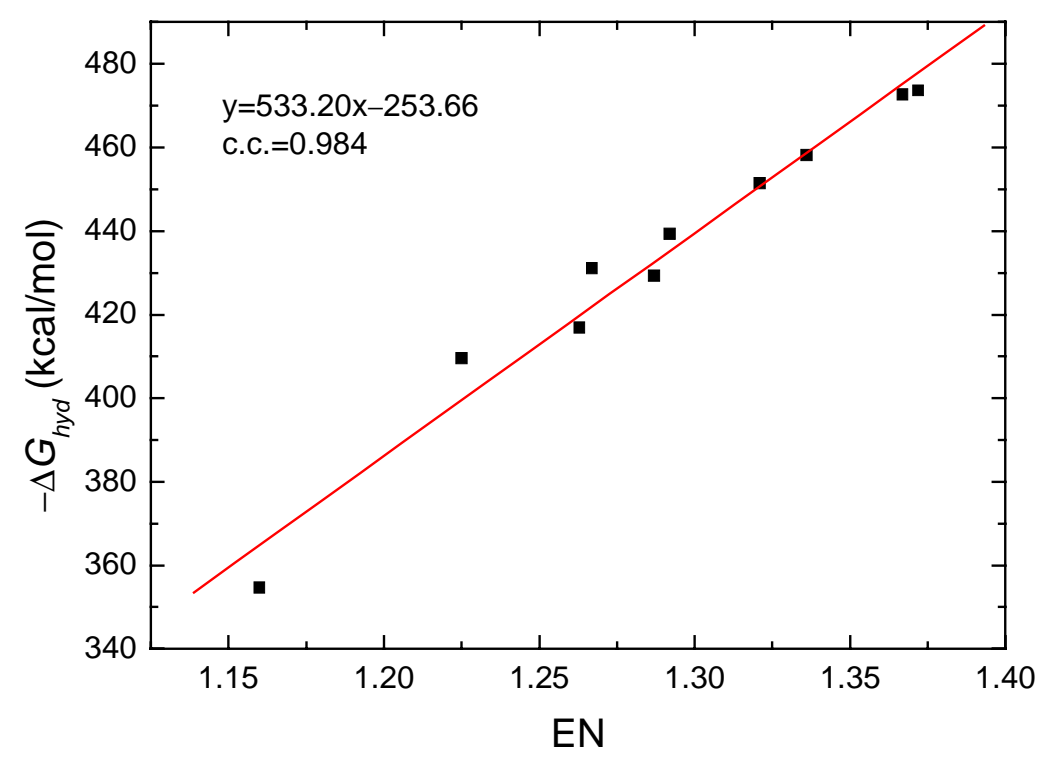

Figure S2. Correlation between negative hydration free energy $\left(-\Delta G_{h y d}\right)$ and $\mathrm{EN}$ of divalent cations for the first transition series (values of EN and the hydration free energy are given in Table S2).

Note: Water is typically a weak-field ligand and a high-spin complex is expected.

Therefore, we choose EN values of $\mathrm{Cr}^{2+}\left(\mathrm{d}^{4}\right), \mathrm{Mn}^{2+}\left(\mathrm{d}^{5}\right), \mathrm{Fe}^{2+}\left(\mathrm{d}^{6}\right)$, and $\mathrm{Co}^{2+}\left(\mathrm{d}^{7}\right)$ in the high-spin states. 
Table S2. The current EN and hydration free energies of divalent metal ions (the hexa-hydrated complex, in the unit of $\mathrm{kcal} / \mathrm{mol}$ ) for the first transition series

\begin{tabular}{ccccccccccc}
\hline Cation & $\mathrm{Ca}^{2+}$ & $\mathrm{Ti}^{2+}$ & $\mathrm{V}^{2+}$ & $\mathrm{Cr}^{2+}$ & $\mathrm{Mn}^{2+}$ & $\mathrm{Fe}^{2+}$ & $\mathrm{Co}^{2+}$ & $\mathrm{Ni}^{2+}$ & $\mathrm{Cu}^{2+}$ & $\mathrm{Zn}^{2+}$ \\
\hline $\mathrm{EN}$ & 1.160 & 1.225 & 1.267 & 1.287 & 1.263 & 1.292 & 1.321 & 1.367 & 1.372 & 1.336 \\
$\Delta G_{h y d}$ & -354.7 & -409.5 & -431.0 & -429.3 & -416.8 & -439.3 & -451.4 & -472.6 & -473.6 & -458.1 \\
\hline
\end{tabular}


Table S3. All the detailed data for calculating EN of six-coordinated cations and our calculated values

\begin{tabular}{|c|c|c|c|c|c|}
\hline Element & $\begin{array}{l}\text { Valence } \\
\text { state }\end{array}$ & $\begin{array}{c}I_{j} \\
(\mathrm{eV})\end{array}$ & $n^{*}$ & $r_{i}$ & $\chi_{i}$ \\
\hline $\mathrm{Li}$ & 1 & 5.392 & 1.99 & 0.9 & 1.009 \\
\hline $\mathrm{Be}$ & 2 & 18.211 & 1.99 & 0.59 & 1.273 \\
\hline B & 3 & 37.933 & 1.99 & 0.41 & 1.714 \\
\hline $\mathrm{C}$ & 4 & 64.486 & 1.99 & 0.3 & 2.380 \\
\hline $\mathrm{N}$ & 3 & 47.447 & 1.99 & 0.3 & 2.164 \\
\hline $\mathrm{N}$ & 5 & 97.838 & 1.99 & 0.27 & 2.939 \\
\hline $\mathrm{O}$ & 6 & 138.115 & 1.99 & 0.23 & 3.758 \\
\hline $\mathrm{F}$ & 7 & 185.177 & 1.99 & 0.22 & 4.368 \\
\hline $\mathrm{Na}$ & 1 & 5.139 & 2.89 & 1.16 & 1.024 \\
\hline $\mathrm{Mg}$ & 2 & 15.035 & 2.89 & 0.86 & 1.234 \\
\hline $\mathrm{Al}$ & 3 & 28.446 & 2.89 & 0.675 & 1.513 \\
\hline $\mathrm{Si}$ & 4 & 45.141 & 2.89 & 0.54 & 1.887 \\
\hline $\mathrm{P}$ & 3 & 30.181 & 2.89 & 0.58 & 1.642 \\
\hline $\mathrm{P}$ & 5 & 65.015 & 2.89 & 0.52 & 2.139 \\
\hline S & 4 & 47.302 & 2.89 & 0.51 & 1.973 \\
\hline$S$ & 6 & 88.044 & 2.89 & 0.43 & 2.659 \\
\hline $\mathrm{Cl}$ & 5 & 67.782 & 2.89 & 0.48 & 2.274 \\
\hline $\mathrm{Cl}$ & 7 & 114.213 & 2.89 & 0.41 & 3.008 \\
\hline $\mathrm{K}$ & 1 & 4.342 & 3.45 & 1.52 & 0.998 \\
\hline $\mathrm{Ca}$ & 2 & 11.867 & 3.45 & 1.14 & 1.160 \\
\hline $\mathrm{Sc}$ & 3 & 24.760 & 3.45 & 0.885 & 1.415 \\
\hline $\mathrm{Ti}$ & 2 & 13.577 & 3.45 & 1 & 1.225 \\
\hline $\mathrm{Ti}$ & 3 & 27.486 & 3.45 & 0.81 & 1.499 \\
\hline $\mathrm{Ti}$ & 4 & 43.271 & 3.45 & 0.745 & 1.730 \\
\hline V & 2 & 14.655 & 3.45 & 0.93 & 1.267 \\
\hline V & 3 & 29.310 & 3.45 & 0.78 & 1.545 \\
\hline V & 4 & 46.711 & 3.45 & 0.72 & 1.795 \\
\hline V & 5 & 65.232 & 3.45 & 0.68 & 2.030 \\
\hline $\mathrm{Cr}$ & $2 \mathrm{~L}$ & 16.500 & 3.45 & 0.87 & 1.322 \\
\hline $\mathrm{Cr}$ & $2 \mathrm{H}$ & 16.500 & 3.45 & 0.94 & 1.287 \\
\hline $\mathrm{Cr}$ & 3 & 30.958 & 3.45 & 0.755 & 1.587 \\
\hline $\mathrm{Cr}$ & 6 & 90.562 & 3.45 & 0.58 & 2.475 \\
\hline Mn & $2 \mathrm{~L}$ & 15.640 & 3.45 & 0.81 & 1.343 \\
\hline $\mathrm{Mn}$ & $2 \mathrm{H}$ & 15.640 & 3.45 & 0.97 & 1.263 \\
\hline $\mathrm{Mn}$ & 4 & 51.199 & 3.45 & 0.67 & 1.912 \\
\hline Mn & 7 & 119.271 & 3.45 & 0.6 & 2.651 \\
\hline $\mathrm{Fe}$ & $2 \mathrm{~L}$ & 16.179 & 3.45 & 0.75 & 1.390 \\
\hline
\end{tabular}




\begin{tabular}{|c|c|c|c|c|c|}
\hline $\mathrm{Fe}$ & $2 \mathrm{H}$ & 16.179 & 3.45 & 0.92 & 1.292 \\
\hline $\mathrm{Fe}$ & $3 \mathrm{~L}$ & 30.647 & 3.45 & 0.69 & 1.651 \\
\hline $\mathrm{Fe}$ & $3 \mathrm{H}$ & 30.647 & 3.45 & 0.785 & 1.556 \\
\hline Co & $2 \mathrm{~L}$ & 17.059 & 3.45 & 0.79 & 1.377 \\
\hline Co & $2 \mathrm{H}$ & 17.059 & 3.45 & 0.885 & 1.321 \\
\hline $\mathrm{Co}$ & $3 \mathrm{~L}$ & 33.497 & 3.45 & 0.685 & 1.693 \\
\hline Co & $3 \mathrm{H}$ & 33.497 & 3.45 & 0.75 & 1.621 \\
\hline $\mathrm{Ni}$ & 2 & 18.168 & 3.45 & 0.83 & 1.367 \\
\hline $\mathrm{Ni}$ & $3 \mathrm{~L}$ & 35.166 & 3.45 & 0.7 & 1.695 \\
\hline $\mathrm{Ni}$ & $3 \mathrm{H}$ & 35.166 & 3.45 & 0.74 & 1.650 \\
\hline $\mathrm{Ni}$ & 4 & 54.930 & 3.45 & 0.62 & 2.037 \\
\hline $\mathrm{Cu}$ & 1 & 7.725 & 3.45 & 0.91 & 1.163 \\
\hline $\mathrm{Cu}$ & 2 & 20.293 & 3.45 & 0.87 & 1.372 \\
\hline $\mathrm{Zn}$ & 2 & 17.964 & 3.45 & 0.88 & 1.336 \\
\hline $\mathrm{Ga}$ & 3 & 30.709 & 3.45 & 0.76 & 1.579 \\
\hline $\mathrm{Ge}$ & 2 & 15.930 & 3.45 & 0.87 & 1.314 \\
\hline $\mathrm{Ge}$ & 4 & 45.706 & 3.45 & 0.67 & 1.854 \\
\hline As & 3 & 28.346 & 3.45 & 0.72 & 1.589 \\
\hline As & 5 & 62.620 & 3.45 & 0.6 & 2.159 \\
\hline $\mathrm{Se}$ & 4 & 42.949 & 3.45 & 0.64 & 1.869 \\
\hline $\mathrm{Se}$ & 6 & 81.701 & 3.45 & 0.56 & 2.448 \\
\hline $\mathrm{Br}$ & 5 & 59.698 & 3.45 & 0.61 & 2.107 \\
\hline $\mathrm{Br}$ & 7 & 103.020 & 3.45 & 0.53 & 2.744 \\
\hline $\mathrm{Rb}$ & 1 & 4.177 & 3.85 & 1.66 & 0.998 \\
\hline $\mathrm{Sr}$ & 2 & 11.030 & 3.85 & 1.32 & 1.139 \\
\hline $\mathrm{Y}$ & 3 & 20.521 & 3.85 & 1.04 & 1.340 \\
\hline $\mathrm{Zr}$ & 4 & 34.337 & 3.85 & 0.86 & 1.610 \\
\hline $\mathrm{Nb}$ & 3 & 25.040 & 3.85 & 0.86 & 1.501 \\
\hline $\mathrm{Nb}$ & 4 & 38.296 & 3.85 & 0.82 & 1.690 \\
\hline $\mathrm{Nb}$ & 5 & 50.546 & 3.85 & 0.78 & 1.862 \\
\hline Mo & 4 & 46.432 & 3.85 & 0.79 & 1.808 \\
\hline Mo & 5 & 61.149 & 3.85 & 0.75 & 2.006 \\
\hline Mo & 6 & 67.989 & 3.85 & 0.73 & 2.101 \\
\hline $\mathrm{Tc}$ & 4 & 42.493 & 3.85 & 0.785 & 1.773 \\
\hline $\mathrm{Tc}$ & 5 & 59.076 & 3.85 & 0.74 & 2.002 \\
\hline $\mathrm{Tc}$ & 7 & 94.314 & 3.85 & 0.7 & 2.384 \\
\hline $\mathrm{Ru}$ & 3 & 28.470 & 3.85 & 0.82 & 1.576 \\
\hline $\mathrm{Ru}$ & 4 & 46.639 & 3.85 & 0.76 & 1.848 \\
\hline $\mathrm{Ru}$ & 5 & 63.222 & 3.85 & 0.705 & 2.099 \\
\hline $\mathrm{Rh}$ & 3 & 31.062 & 3.85 & 0.805 & 1.622 \\
\hline $\mathrm{Rh}$ & 4 & 45.602 & 3.85 & 0.74 & 1.863 \\
\hline $\mathrm{Rh}$ & 5 & 67.367 & 3.85 & 0.69 & 2.167 \\
\hline $\mathrm{Pd}$ & 2 & 19.433 & 3.85 & 1 & 1.346 \\
\hline $\mathrm{Pd}$ & 3 & 32.927 & 3.85 & 0.9 & 1.562 \\
\hline
\end{tabular}




\begin{tabular}{|c|c|c|c|c|c|}
\hline $\mathrm{Pd}$ & 4 & 48.712 & 3.85 & 0.755 & 1.876 \\
\hline $\mathrm{Ag}$ & 1 & 7.576 & 3.85 & 1.29 & 1.097 \\
\hline $\mathrm{Ag}$ & 2 & 21.485 & 3.85 & 1.08 & 1.333 \\
\hline $\mathrm{Cd}$ & 2 & 16.904 & 3.85 & 1.09 & 1.276 \\
\hline In & 3 & 28.025 & 3.85 & 0.94 & 1.480 \\
\hline $\mathrm{Sn}$ & 2 & 14.632 & 3.85 & 1.32 & 1.181 \\
\hline $\mathrm{Sn}$ & 4 & 40.733 & 3.85 & 0.83 & 1.706 \\
\hline $\mathrm{Sb}$ & 3 & 25.320 & 3.85 & 0.9 & 1.476 \\
\hline $\mathrm{Sb}$ & 5 & 55.967 & 3.85 & 0.74 & 1.971 \\
\hline $\mathrm{Te}$ & 4 & 37.415 & 3.85 & 1.11 & 1.467 \\
\hline $\mathrm{Te}$ & 6 & 70.705 & 3.85 & 0.7 & 2.180 \\
\hline I & 5 & 51.821 & 3.85 & 1.09 & 1.587 \\
\hline $\mathrm{I}$ & 7 & 90.169 & 3.85 & 0.67 & 2.417 \\
\hline $\mathrm{Cs}$ & 1 & 3.894 & 4.36 & 1.81 & 0.998 \\
\hline $\mathrm{Ba}$ & 2 & 10.002 & 4.36 & 1.49 & 1.126 \\
\hline $\mathrm{La}$ & 3 & 19.174 & 4.36 & 1.172 & 1.327 \\
\hline Hf & 4 & 33.331 & 4.36 & 0.85 & 1.706 \\
\hline $\mathrm{Ta}$ & 3 & 21.765 & 4.36 & 0.86 & 1.536 \\
\hline $\mathrm{Ta}$ & 4 & 33.165 & 4.36 & 0.82 & 1.735 \\
\hline $\mathrm{Ta}$ & 5 & 44.566 & 4.36 & 0.78 & 1.925 \\
\hline $\mathrm{W}$ & 4 & 35.238 & 4.36 & 0.8 & 1.784 \\
\hline $\mathrm{W}$ & 5 & 47.675 & 4.36 & 0.76 & 1.991 \\
\hline $\mathrm{W}$ & 6 & 61.149 & 4.36 & 0.74 & 2.175 \\
\hline $\mathrm{Re}$ & 4 & 37.726 & 4.36 & 0.77 & 1.853 \\
\hline $\mathrm{Re}$ & 6 & 65.294 & 4.36 & 0.69 & 2.317 \\
\hline $\mathrm{Re}$ & 7 & 78.768 & 4.36 & 0.67 & 2.507 \\
\hline Os & 4 & 40.420 & 4.36 & 0.77 & 1.888 \\
\hline Os & 6 & 68.404 & 4.36 & 0.685 & 2.362 \\
\hline Os & 7 & 83.950 & 4.36 & 0.665 & 2.573 \\
\hline Ir & 3 & 26.947 & 4.36 & 0.82 & 1.649 \\
\hline Ir & 4 & 39.384 & 4.36 & 0.765 & 1.881 \\
\hline $\mathrm{Ir}$ & 5 & 57.003 & 4.36 & 0.71 & 2.183 \\
\hline $\mathrm{Pt}$ & 2 & 18.562 & 4.36 & 0.94 & 1.432 \\
\hline $\mathrm{Pt}$ & 4 & 40.420 & 4.36 & 0.765 & 1.895 \\
\hline $\mathrm{Pt}$ & 5 & 54.930 & 4.36 & 0.71 & 2.159 \\
\hline $\mathrm{Au}$ & 1 & 9.225 & 4.36 & 1.51 & 1.113 \\
\hline $\mathrm{Au}$ & 3 & 30.056 & 4.36 & 0.99 & 1.550 \\
\hline $\mathrm{Au}$ & 5 & 58.040 & 4.36 & 0.71 & 2.195 \\
\hline $\mathrm{Hg}$ & 1 & 10.437 & 4.36 & 1.33 & 1.165 \\
\hline $\mathrm{Hg}$ & 2 & 18.756 & 4.36 & 1.16 & 1.326 \\
\hline $\mathrm{Tl}$ & 1 & 6.108 & 4.36 & 1.64 & 1.050 \\
\hline $\mathrm{Tl}$ & 3 & 29.828 & 4.36 & 1.025 & 1.524 \\
\hline $\mathrm{Pb}$ & 2 & 15.032 & 4.36 & 1.33 & 1.225 \\
\hline $\mathrm{Pb}$ & 4 & 42.317 & 4.36 & 0.915 & 1.746 \\
\hline
\end{tabular}




\begin{tabular}{cccccc}
\hline $\mathrm{Bi}$ & 3 & 25.558 & 4.36 & 1.17 & 1.399 \\
$\mathrm{Bi}$ & 5 & 55.967 & 4.36 & 0.9 & 1.895 \\
$\mathrm{Po}$ & 4 & 38.348 & 4.36 & 1.08 & 1.575 \\
$\mathrm{Po}$ & 6 & 72.549 & 4.36 & 0.81 & 2.168 \\
$\mathrm{At}$ & 7 & 91.205 & 4.36 & 0.76 & 2.423 \\
$\mathrm{Fr}$ & 1 & 4.146 & 4.99 & 1.94 & 1.012 \\
$\mathrm{Ra}$ & 2 & 10.147 & 4.99 & 1.57 & 1.151 \\
$\mathrm{Ac}$ & 3 & 19.692 & 4.99 & 1.26 & 1.363 \\
$\mathrm{Ce}$ & 3 & 20.200 & 4.36 & 1.15 & 1.348 \\
$\mathrm{Ce}$ & 4 & 36.762 & 4.36 & 1.01 & 1.608 \\
$\mathrm{Pr}$ & 3 & 21.620 & 4.36 & 1.13 & 1.374 \\
$\mathrm{Pr}$ & 4 & 38.980 & 4.36 & 0.99 & 1.646 \\
$\mathrm{Nd}$ & 3 & 22.076 & 4.36 & 1.123 & 1.382 \\
$\mathrm{Pm}$ & 3 & 22.283 & 4.36 & 1.11 & 1.391 \\
$\mathrm{Sm}$ & 3 & 23.423 & 4.36 & 1.098 & 1.410 \\
$\mathrm{Eu}$ & 2 & 11.245 & 4.36 & 1.31 & 1.181 \\
$\mathrm{Eu}$ & 3 & 24.916 & 4.36 & 1.087 & 1.433 \\
$\mathrm{Gd}$ & 3 & 20.625 & 4.36 & 1.078 & 1.386 \\
$\mathrm{~Tb}$ & 3 & 21.910 & 4.36 & 1.063 & 1.410 \\
$\mathrm{~Tb}$ & 4 & 39.788 & 4.36 & 0.9 & 1.733 \\
$\mathrm{Dy}$ & 2 & 11.670 & 4.36 & 1.21 & 1.213 \\
$\mathrm{Dy}$ & 3 & 22.801 & 4.36 & 1.052 & 1.426 \\
$\mathrm{Ho}$ & 3 & 22.843 & 4.36 & 1.041 & 1.433 \\
$\mathrm{Er}$ & 3 & 22.739 & 4.36 & 1.03 & 1.438 \\
$\mathrm{Tm}$ & 2 & 12.054 & 4.36 & 1.17 & 1.231 \\
$\mathrm{Tm}$ & 3 & 23.682 & 4.36 & 1.02 & 1.455 \\
$\mathrm{Yb}$ & 2 & 12.188 & 4.36 & 1.16 & 1.237 \\
$\mathrm{Yb}$ & 3 & 25.030 & 4.36 & 1.008 & 1.479 \\
$\mathrm{Lu}$ & 3 & 20.956 & 4.36 & 1.001 & 1.431 \\
\hline & & & & &
\end{tabular}

Note: Data of the ionization energy in Ref. 19 are given in the unit of $\mathrm{kJ} / \mathrm{mol}$. To convert such a unit from $\mathrm{kJ} / \mathrm{mol}$ to $\mathrm{eV}$ is to divide its value by 96.486 . 


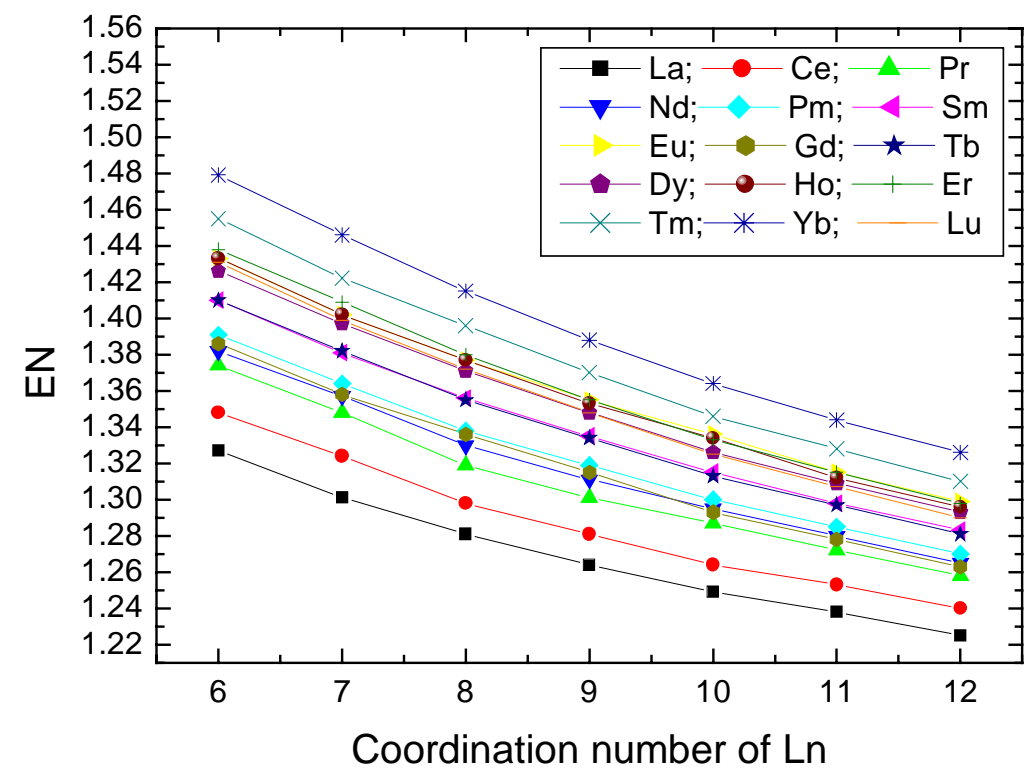

Figure S3. EN of trivalent $\mathrm{Ln}$ as a function of $\mathrm{CN}$. 


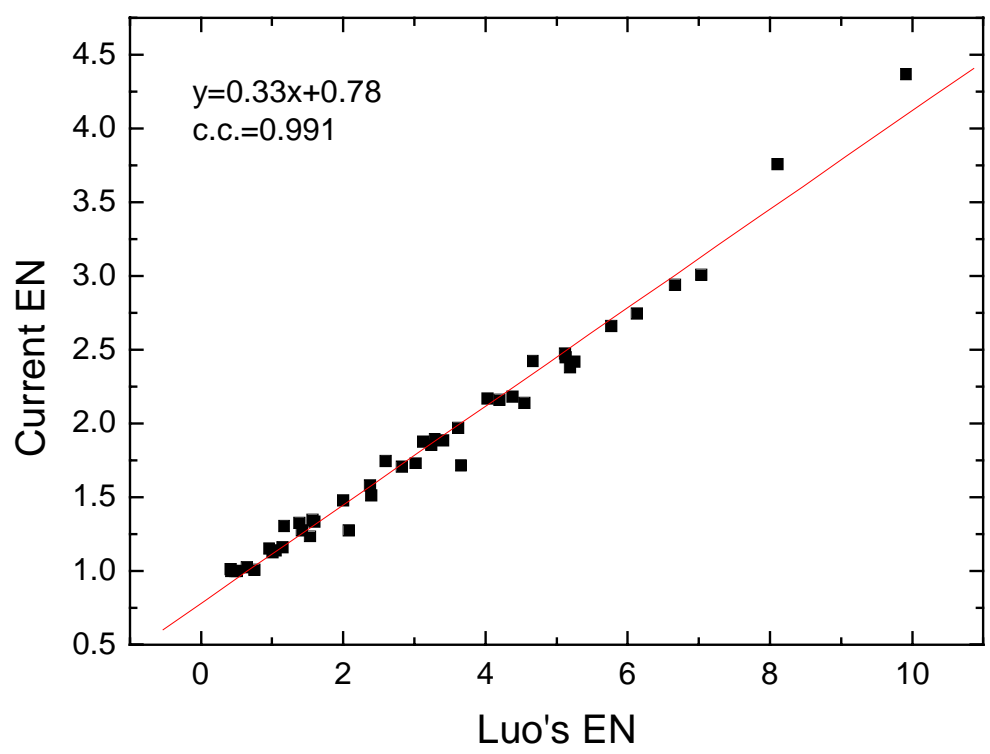

Figure S4. A fairly good relation between our EN scale and Luo's EN scale, which is on the basis of covalent potential (values of current EN and Luo's EN are given in Table S4). 
Table S4. The current EN and Luo's EN

\begin{tabular}{cccccc}
\hline $\begin{array}{c}\text { Element } \\
\text { (valence) }\end{array}$ & Current EN & Luo's EN & $\begin{array}{c}\text { Element } \\
\text { (valence) }\end{array}$ & Current EN & Luo's EN \\
\hline $\mathrm{Li}(1)$ & 1.009 & 0.75 & $\mathrm{P}(5)$ & 2.139 & 4.55 \\
$\mathrm{Na}(1)$ & 1.024 & 0.65 & $\mathrm{As}(5)$ & 2.159 & 4.20 \\
$\mathrm{~K}(1)$ & 0.998 & 0.51 & $\mathrm{Sb}(5)$ & 1.971 & 3.62 \\
$\mathrm{Rb}(1)$ & 0.998 & 0.48 & $\mathrm{Bi}(5)$ & 1.895 & 3.29 \\
$\mathrm{Cs}(1)$ & 0.998 & 0.43 & $\mathrm{O}(6)$ & 3.758 & 8.11 \\
$\mathrm{Fr}(1)$ & 1.012 & 0.42 & $\mathrm{~S}(6)$ & 2.659 & 5.77 \\
$\mathrm{Be}(2)$ & 1.273 & 2.08 & $\mathrm{Se}(6)$ & 2.448 & 5.13 \\
$\mathrm{Mg}(2)$ & 1.234 & 1.54 & $\mathrm{Te}(6)$ & 2.180 & 4.38 \\
$\mathrm{Ca}(2)$ & 1.160 & 1.15 & $\operatorname{Po}(6)$ & 2.168 & 4.03 \\
$\mathrm{Sr}(2)$ & 1.139 & 1.05 & $\mathrm{~F}(7)$ & 4.368 & 9.915 \\
$\mathrm{Ba}(2)$ & 1.126 & 1.01 & $\mathrm{Cl}(7)$ & 3.008 & 7.04 \\
$\mathrm{Ra}(2)$ & 1.151 & 0.96 & $\mathrm{Br}(7)$ & 2.744 & 6.13 \\
$\mathrm{~B}(3)$ & 1.714 & 3.66 & $\mathrm{I}(7)$ & 2.417 & 5.25 \\
$\mathrm{Al}(3)$ & 1.513 & 2.40 & $\mathrm{At}(7)$ & 2.423 & 4.67 \\
$\mathrm{Ga}(3)$ & 1.579 & 2.38 & $\operatorname{Ti}(4)$ & 1.730 & 3.02 \\
$\mathrm{In}(3)$ & 1.480 & 2.00 & $\mathrm{Cr}(6)$ & 2.475 & 5.12 \\
$\mathrm{C}(4)$ & 2.380 & 5.19 & $\mathrm{Cr}(2)$ & 1.305 & 1.171 \\
$\mathrm{Si}(4)$ & 1.887 & 3.41 & $\mathrm{Zn}(2)$ & 1.336 & 1.60 \\
$\mathrm{Ge}(4)$ & 1.854 & 3.24 & $\mathrm{Cd}(2)$ & 1.276 & 1.42 \\
$\mathrm{Sn}(4)$ & 1.706 & 2.83 & $\mathrm{Hg}(2)$ & 1.326 & 1.39 \\
$\mathrm{~Pb}(4)$ & 1.746 & 2.60 & $\operatorname{Pd}(4)$ & 1.876 & 3.13 \\
$\mathrm{~N}(5)$ & 2.939 & 6.67 & $\operatorname{Pd}(2)$ & 1.346 & 1.57 \\
\hline & & & & &
\end{tabular}

